BULL. AUSTRAL. MATH. SOC.

VOL. 16 (1977), 61-65.

\title{
Pseudo-autonomous linear systems
}

\section{W.A. Coppel}

\begin{abstract}
Pseudo-autonomous linear differential equations are defined. A linear differential equation with bounded coefficient matrix is pseudo-autonomous if and only if it is almost reducible. A linear differential equation with recurrent coefficient matrix is pseudo-autonomous if and only if it has pure point spectrum.
\end{abstract}

Let $X(t)$ be the fundamental matrix for the linear differential equation

$$
x^{\prime}=A(t) x
$$

such that $X(0)=I$, where the $n \times n$ coefficient matrix $A(t)$ is continuous on the whole line $-\infty<t<\infty$. The equation ( 1 ) will be said to be pseudo-autonomous if there exist real numbers $\lambda_{1}, \ldots, \lambda_{m}$ and supplementary projections $P_{1}, \ldots, P_{m}$ such that for each $\varepsilon>0$ there is a constant $K_{\varepsilon}>0$ satisfying

$$
\left|X(t) P_{j} X^{-1}(s)\right| \leq K_{\varepsilon} e^{\lambda_{j}(t-s)} e^{\varepsilon|t-s|}
$$

for $-\infty<s, t<\infty$, and $j=1, \ldots, m$.

It follows that, for any non-trivial solution $x(t)$ of (I), $P_{j} x(0)=x(0)$ implies

$$
\lim _{t \rightarrow \pm \infty} t^{-1} \log |x(t)|=\lambda_{j}
$$

Hence the numbers $\lambda_{j}$ and the projections $P_{j}$ are uniquely determined. Moreover, the Lyapunov characteristic exponents of $(1)$ are $\lambda_{1}, \ldots, \lambda_{m}$ Received 23 August 1976. 
with multiplicity $\operatorname{tr} P_{1}, \ldots$, tr $P_{m}$ respectively.

If (1) is pseudo-autonomous then by [2, Lemma 2], it is kinematically similar to a block diagonal system

$$
y_{j}^{\prime}=B_{j}(t) y_{j} \quad(j=1, \ldots, m)
$$

whose fundamental matrices $Y_{j}(t)$ satisfy

$$
\left|Y_{j}(t) Y_{j}^{-1}(s)\right| \leq L_{\varepsilon} e^{\lambda_{j}(t-s)} e^{\varepsilon|t-s|}
$$

for $-\infty<s, t<\infty$, and $j=1, \ldots, m$. Moreover, if $A(t)$ is bounded then $B_{1}(t), \ldots, B_{m}(t)$ are also bounded.

Pseudo-autonomous equations are closely connected to the almost reducible equations of Bylov [1]. In fact, if (1) is almost reducible then by $[1$, Corollary $2, p .344]$ there exist real numbers $\lambda_{1}, \ldots, \lambda_{m}$ and positive integers $n_{1}, \ldots, n_{m}$ with sum $n$ such that, for every $\varepsilon>0$, (1) is kinematically similar to an equation

$$
z^{\prime}=[C+D(t)] z
$$

where $C=\operatorname{diag}\left[\lambda_{1} I_{n}, \ldots, \lambda_{m} I_{n}\right]$ and $|D(t)| \leq \varepsilon$ for $-\infty<t<\infty$. Since by the proof of [2, Theorem 2] we can assume further that $D(t)=\operatorname{diag}\left[D_{1}(t), \ldots, D_{m}(t)\right]$, it follows that $(1)$ is pseudo-autonomous.

Conversely, if ( 1 ) is pseudo-autonomous and its coefficient matrix $A(t)$ is bounded then it is almost reducible. To see this it is sufficient to show that if an equation

$$
y^{\prime}=B(t) y
$$

with bounded coefficient matrix $B(t)$ has a fundamental matrix $Y(t)$ satisfying

$$
\left|Y(t) Y^{-1}(s)\right| \leq L_{\varepsilon} e^{\varepsilon|t-s|}
$$

then it is almost reducible to 0 . We have

$$
\left|\operatorname{det} Y(t) Y^{-1}(s)\right| \leq M_{\varepsilon} e^{n \varepsilon|t-s|}
$$


and hence, by Liouville's formula,

$$
\left|R \int_{s}^{t} \operatorname{tr} B(\tau) d \tau\right| \leq \log M_{\varepsilon}+n \varepsilon|t-s| .
$$

Thus

$$
\lim _{t-s \rightarrow \infty}(t-s)^{-1} \int_{s}^{t} R \operatorname{tr} B(\tau) d \tau=0,
$$

and the result follows from [1, Theorem 7$]$.

Assume now that the coefficient matrix $A(t)$ is bounded and uniformly continuous. Then by Ascoli's Theorem any sequence $\left\{h_{\nu}\right\}$ of real numbers contains a subsequence $\left\{k_{v}\right\}$ such that

$$
\tilde{A}(t)=\lim _{v \rightarrow \infty} A(t+k)
$$

exists uniformly on compact intervals. The collection of all equations

$$
x^{\prime}=\tilde{A}(t) x
$$

is called the hull of the equation (1). The fundamental matrix $\tilde{X}(t)$ of (2) such that $\tilde{X}(0)=I$ is given by

$$
\tilde{X}(t)=\lim _{\nu \rightarrow \infty} X\left(t+k_{\nu}\right) X^{-1}\left(k_{\nu}\right)
$$

Suppose (1) is pseudo-autonomous. Then, by restricting attention in the first instance to a subsequence, $X\left(k_{v}\right) P_{j} X^{-1}\left(k_{v}\right) \rightarrow \tilde{P}_{j}$, where $\tilde{P}_{1}, \ldots, \tilde{P}_{m}$ are supplementary projections. It follows that

$$
\left|\tilde{X}(t) \tilde{P}_{j} \tilde{X}^{-1}(s)\right| \leq K_{\varepsilon} e^{\lambda_{j}(t-s)} e^{\varepsilon|t-s|}
$$

for $-\infty<s, t<\infty$, and $j=1, \ldots, m$. Thus every equation (2) in the hull of (1) is also pseudo-autonomous, with the same numbers $\lambda_{j}$ and similar projections $\tilde{P}_{j}$. Hence all equations in the hull of a pseudoautonomous equation have the same characteristic exponents, counting multiplicities. Moreover, if (2) has a non-trivial solution $\tilde{x}(t)$ such that $e^{-\lambda t}|\tilde{x}(t)|$ is bounded on $-\infty<t<\infty$ for some real $\lambda$, then 
$\lambda=\lambda_{j}$ for some $j$. In fact, $j$ is the greatest integer $k$ for which $\tilde{P}_{k} \tilde{x}(0) \neq 0$. Therefore a pseudo-autonomous equation has pure point spectrum, in the terminology of Sacker and Sell [6].

If $A(t)$ is recurrent and (1) has pure point spectrum then conversely (1) is pseudo-autonomous, by Sacker and Sell [6, Theorem 2].

Suppose finally that $A(t)$ is almost periodic and all equations (2) in the hull of ( 1 ) have the same characteristic exponents, counting multiplicities. Then the sum of the characteristic exponents is the same for all equations (2). It follows that if some equation (2) is regular, in the sense of Lyapunov, then every equation (2) is regular, since $\operatorname{tr} \tilde{A}(t)$ has the same mean value as $\operatorname{tr} A(t)$. But according to Millionščikov [4, Theorem 3], at least one equation in the hull is regular. Hence all equations in the hull of (I) are regular. Therefore (I) is almost reducible, by Millionščikov [3, Theorem 1 and Lemma] (cf. the proof of Theorem 2).

The preceding results establish in particular the

THEOREM. If the coefficient matrix $A(t)$ is almost periodic then the following assertions are equivalent:

(i) the equation (1) is pseudo-autonomous;

(ii) the equation (1) is almost reducible;

(iii) the equation (1) has pure point spectrom;

(iv) all equations in the hull of (1) have the same characteristic exponents.

Millionšcikov [5] has given an example of an equation (1) which is not almost reducible, even though $A(t)$ is quasi-periodic. In conjunction with the theorem, this disproves Sacker and Sell's conjecture that every almost periodic linear differential system has pure point spectrum. 


\section{References}

[1] Б.Ф. Былов [B.F. Bylov], "Почти приводимые системы днфференциальных уравнений" [A]most reducible systems of differential equations], Sibirsk. Mat. Ž. 3 (1962), 333-359.

[2] W.A. Coppel, "Dichotomies and reducibility", J. Differential Equations 3 (1967), 500-521.

[3] В.M. Миллионщинов [V.M. Millionščikov], "О связи мењду устойчнвостью харантеристичесних покаэателей и почти прнводимостью снстем с почти периодичесними ноэффнциентами" [The connection between the stability of characteristic exponents and almost reducibility of systems with almost periodic coefficients], Differencial'nye Uravnenija 3 (1967), 2127-2134.

[4] В.M. Миллионщинов [V.M. Millionščikov], "Нритернй устойчивости вероятного спентра линейных систем дифференциальных уравнений с ренуррентными ноэффициентами и нритернй почти приводимости систем с почти периодичесними ноэффициентами" [A criterion for the stability of the probable spectrum of linear systems of differential equations with recurrent coefficients and a criterion for the almost reducibility of systems with almost periodic coefficients], Mat. Sb. (N.S.) 78 (1969), 179-201.

[5] В.М. Мнллионщинов [V.M. Millionščikov]. "Доназательство существовання неправильных снстем линейных дифференцнальных уравнений с нваэипериодичесннмн ноэффициентамн" [A proof of the existence of irregular systems of linear differential equations with quasiperiodic coefficients], Differencial'nye Uravnenija 5 (1969). 1979-1983.

[6] Robert J. Sacker and George R. Sell, "A spectral theory for linear almost periodic differential equations", International Conference on Differential Equations, 698-708 (Proc. Internat. Conf. Differential Equations, Los Angeles, California, 1974. Academic Press [Harcourt Brace Jovanovich], New York, London, 1975).

Department of Mathematics, Institute of Advanced Studies, Australian National University, Canberra, ACT. 